\title{
Uso de plasma convaleciente en COVID-19
}

\section{J. David Macedo-Reynada ${ }^{*}$ y Yanet Ventura-Enríquez ${ }^{2}$}

${ }^{1}$ Banco de Células Troncales Hematopoyéticas, Banco Central de Sangre, Centro Médico Nacional La Raza; '2Departamento de Banco de Sangre, Centro Médico Naval. Ciudad de México, México

\section{Resumen}

En el momento actual no existen opciones terapéuticas específicas o vacuna para la enfermedad por coronavirus 2019 (COVID-19), causada por el coronavirus 2 del síndrome respiratorio agudo grave (SARS-CoV-2). La opción con plasma convaleciente resulta atractiva, dados sus precedentes en pandemias previas, su rápida disponibilidad y su bien conocido perfil de bioseguridad. Se propone la eficacia del uso de inmunidad pasiva por medio de la transfusión de plasma convaleciente mediante distintos mecanismos de acción (neutralización, citotoxicidad mediada por anticuerpos, activación del complemento, inmunomodulación). El uso de plasma convaleciente está aprobado en pacientes con datos de gravedad o criterios de complicabilidad. Los resultados disponibles de experiencias internacionales y locales demuestran un adecuado perfil de bioseguridad $<1 \%$ de eventos adversos graves reportados). La evidencia de la eficacia de la intervención es limitada a no más de seis series de casos, destacando la necesidad de una administración temprana, un seguimiento estrecho de variables de desenlace y la comunicación de series más robustas y con una mayor fortaleza metodológica. En nuestro país diversos grupos se encuentran realizando esfuerzos colaborativos y/o metacéntricos, lo que nos ayudará a dilucidar la relevancia de esta intervención en un panorama aún complejo de una epidemia que se encuentra en su cénit sin que se cuente con herramientas específicas para su tratamiento.

PALABRAS CLAVE: Plasma convaleciente. SARS-CoV-2. Inmunidad pasiva. Tratamiento. COVID-19.

\section{Use of convalescent plasma in COVID-19}

\section{Abstract}

At the present time, there are no specific therapeutic options or vaccine for coronavirus disease 2019 (COVID-19), produced by the severe acute respiratory syndrome coronavirus 2 (SARS-CoV-2). The convalescent plasma option is attractive given its precedents in the context of previous pandemics, its rapid availability in areas where the epidemic is active, and its well-known biosecurity profile. There is emerging evidence that proposes the effectiveness of the use of passive immunity through convalescent plasma transfusion, which would exert its effect through different mechanisms of action (neutralization, antibody-mediated cytotoxicity, complement activation, immunomodulation). Globally, the use of convalescent plasma is approved in patients with severe disease criteria or high risk of complication. The available results of international and local experiences demonstrate an adequate biosecurity profile of the use of convalescent plasma with a rate of serious adverse events related to the intervention of less than $1 \%$. So far, the evidence for the efficacy of the intervention is limited to no more than 6 case series, highlighting the need for early administration, close monitoring of outcome variables, and communication of more robust series with greater methodological strength. In our country, several groups are making collaborative and/or metacentric efforts, which will help us to clarify the relevance of this intervention in a still complex panorama of an epidemic that is its highest point without specific tools for its treatment.

KEY WORDS: Convalescent plasma. SARS-CoV-2. Passive immunity. COVID-19.

\footnotetext{
Correspondencia:

*J. David Macedo-Reynada

E-mail: dave16z89@gmail.com

0016-3813/๑ 2021 Academia Nacional de Medicina de México, A.C. Publicado por Permanyer. Este es un artículo open access bajo la licencia CC BY-NC-ND (http://creativecommons.org/licenses/by-nc-nd/4.0/).

Fecha de recepción: 09-09-2020

Fecha de aceptación: 09-02-202

DOI: 10.24875/GMM.M21000476

Gac Med Mex. 2021;157(Supl 3):S68-S78

Disponible en PubMed

www.gacetamedicademexico.com
} 


\section{Introducción}

La epidemia de enfermedad por coronavirus 2019 (COVID-19), causada por el coronavirus 2 del síndrome respiratorio agudo grave (SARS-CoV-2), se originó en diciembre de 2019 en Wuhan, China'. El 30 de enero de 2020, la Organización Mundial de la Salud (OMS) declara que el brote de la infección por el coronavirus SARS-CoV-2 (COVID-19) constituye una emergencia de salud pública de importancia internacional. El 11 de marzo de 2020, la OMS declara oficialmente la pandemia, la cual se ha extendido de manera global, con un acumulado de casi 9 millones de casos y 500,000 fallecimientos ${ }^{2}$.

En el momento actual, no existen opciones terapéuticas específicas o vacuna para la COVID-19. Se encuentran bajo investigación terapias como remdesivir (antiviral que disminuye la producción de ARN viral ${ }^{3,4}$, favipiravir (antiviral análogo de nucleósidos que inhibe la síntesis de la cadena de ARN) ${ }^{5,6}$, hidroxicloroquina/cloroquina (limita la replicación del SARS-CoV-2) ${ }^{7}$, hidroxicloroquina/azitromicina (favorece la eliminación viral) ${ }^{8}$, lopinavir/ritonavir (inhibe la actividad proteasa del coronavirus) ${ }^{9}$, anticuerpos monoclonales (tocilizumab, antagonista del receptor de la interleucina [IL] 6) ${ }^{10}$ y plasma de donadores convalecientes (inmunoterapia pasiva) ${ }^{11}$.

La opción con plasma convaleciente resulta atractiva, dados sus precedentes en el contexto de pandemias previas, su rápida disponibilidad en zonas donde la epidemia se encuentre activa y su bien conocido perfil de bioseguridad.

\section{Antecedentes históricos del uso de plasma convaleciente}

A inicios del siglo $x x$, el plasma convaleciente fue utilizado como una intervención de inmunidad pasiva para el tratamiento en brotes epidémicos de infecciones virales como poliomielitis ${ }^{12}$, varicela ${ }^{13,14}$, parotiditis $^{15}$ e influenza ${ }^{16}$. Durante la pandemia de influenza (1918-1925) se evaluó el uso de componentes sanguíneos en el tratamiento hospitalario de neumonía viral vs. un grupo control. Un metaanálisis realizado casi un siglo después mostró una reducción de la tasa cruda de fatalidad del $37 \%$ en los controles a un $16 \%$ en los pacientes que recibieron la intervención. El beneficio se vio maximizado si estos recibían el tratamiento en los primeros cinco días de la aparición de las complicaciones por neumonía ${ }^{17}$. A pesar de las limitaciones de dicho estudio (no cegado, no aleatorizado, sin control con placebo), se destacó el potencial beneficio del plasma convaleciente en la era moderna, por lo que cobró nuevo interés con el surgimiento de nuevas pandemias. El uso de plasma convaleciente fue recomendado como tratamiento empírico de SARS-CoV, coronavirus causante del síndrome respiratorio de Oriente Medio (MERS-CoV), influenza aviar H5N1 e influenza H1N1 y durante el brote por el virus del Ébola en el año 2014 ${ }^{18-21}$. En un estudio en el que se incluyeron 99 pacientes con influenza pandémica A-H1N1 en 2009, se observó que en los 20 pacientes en estado crítico que recibieron plasma convaleciente existió una reducción en la mortalidad (odds ratio [OR]: 0.20; intervalo de confianza del 95\% [IC 95\%]: 0.06-0.69; $p=0.011$ ) e incluso disminución de la carga viral y respuesta sérica de citocinas (IL-6, IL19, factor de necrosis tumoral $\alpha)^{22}$. Estudios descriptivos sugieren permite reducir la estancia hospitalaria y la mortalidad (sobre todo cuando se administra de manera temprana) y es suficientemente segura ${ }^{23,24}$. En el caso del MERS los hallazgos previos han sugerido el uso de plasma convaleciente ante situaciones clínicas en las que no existen vacunas o medicamentos específicos ${ }^{25,26}$.

\section{Uso de plasma convaleciente que la administración de plasma convaleciente en pacientes con SARS en COVID-19: evidencia disponible (experiencia internacional)}

De acuerdo con una revisión sistemática que evaluó la experiencia clínica de China con la administración de plasma convaleciente, hasta el 5 de mayo de 2020 en ese país se habían recogido 2,000 muestras de plasma convaleciente, administrándose a un total de al menos 700 pacientes con COVID-1927,28. Según los datos reportados en esta revisión, se recolectaron entre 200-400 ml de plasma en cada donación, con títulos de anticuerpos $>1: 160$. Al revisar toda la evidencia clínica generada hasta ese momento, fueron identificados y analizados tres estudios clínicos que evaluaban a 19 pacientes que recibieron terapia con plasma convaleciente ${ }^{29-31}$.

Los detalles de estos tres estudios (un estudio piloto, una serie de casos y un informe de caso) se presentan en la tabla 1. En los tres, la mediana de edad fue de 55 años (rango intercuartílico [IQR]: 46-67 años) la edad de los pacientes fue de 30 a 78 años. La mediana del tiempo desde el inicio de los síntomas hasta la admisión 


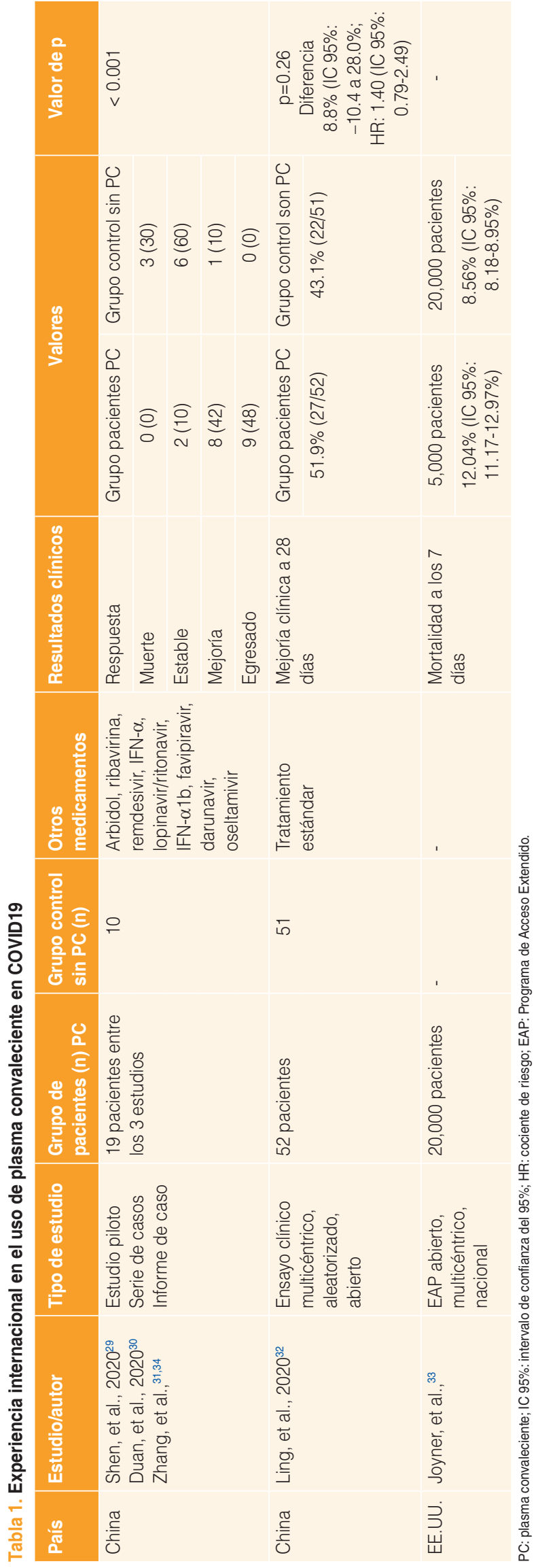

y la transfusión de plasma convaleciente fue de 4 días (IQR: 2-8 días) y 19 días (IQR: 14-20 días), respectivamente. Siete pacientes tenían enfermedades crónicas (enfermedades cardiovasculares y/o cerebrovasculares, insuficiencia renal crónica, enfermedad pulmonar obstructiva crónica e hipertensión). Antes de la terapia con plasma convaleciente, 12 pacientes recibieron esteroides (metilprednisolona) y todos los pacientes recibieron algún antiviral, como terapia convencional, 12 pacientes se encontraban con ventilación mecánica, seis con oxígeno por cánula nasal de alto flujo y dos recibieron oxigenación convencional con cánula nasal de bajo flujo.

Se formó un grupo de control histórico mediante una selección aleatoria de 10 pacientes, de la cohorte del estudio de Duan, et al..$^{30}$, que coincidían con la edad, el sexo y la gravedad de la enfermedad. Las características basales de los pacientes entre el grupo de tratamiento con plasma convaleciente y el grupo control se mostraron sin diferencias significativas $(p>0.05)$. La linfopenia, un índice importante en el pronóstico en COVID-19, tendió a mejorar después de transfusión de plasma convaleciente (mediana: $0.65 \times 10^{9} / l$ vs. 0.76 $\times 10 \% /)$. Este análisis mostró una mejora estadísticamente significativa en los resultados clínicos de los pacientes que recibieron plasma en comparación con los casos no tratados $(p<0.001)$. No se informaron efectos adversos graves (Tabla 1).

Ling, et al. publicaron los resultados de su ensayo clínico aleatorizado, que incluyó a 103 pacientes con COVID-19 grave o potencialmente mortal, y reportaron que la terapia de plasma convaleciente agregada al tratamiento estándar no mejoró significativamente el tiempo de mejoría clínica dentro de los 28 días, aunque el ensayo se terminó temprano y puede haber tenido poca potencia para detectar una diferencia clínicamente importante. La razón de riesgo de tiempo para la mejoría clínica dentro de los 28 días en el grupo de pacientes que recibió plasma convaleciente vs. el grupo de tratamiento estándar fue de 1.40 y no fue estadísticamente significativa ${ }^{32}$.

Recientemente, se reportó la actualización de los resultados de seguridad del Programa de Acceso Extendido (EAP), iniciativa de la Food and Drug Administration (FDA ${ }^{33}$, en colaboración con la Clínica Mayo y la comunidad nacional de bancos de sangre. Este ensayo, abierto, multicéntrico y nacional (EE.UU.), incluyó la administración de plasma convaleciente a 20,000 pacientes hospitalizados con COVID-19, habiendo demostrado previamente la seguridad en 5,000 pacientes hospitalizados. La tasa general de mortalidad a los siete días fue del $8.56 \%$ (IC 95\%: 


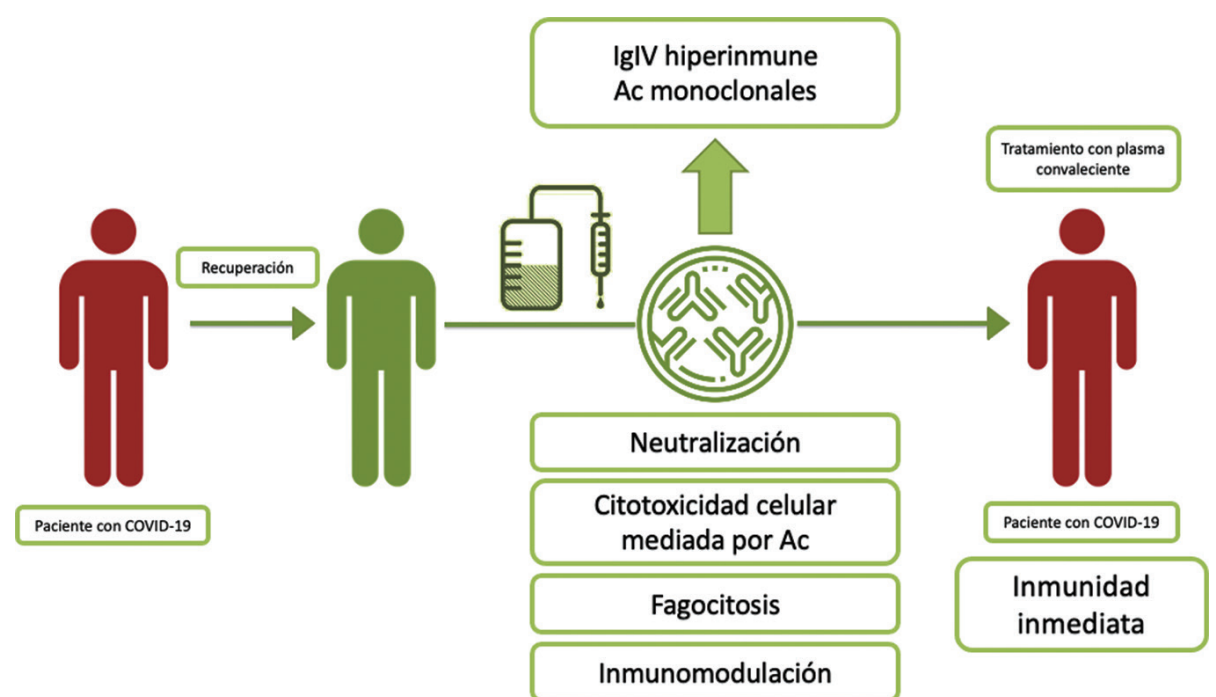

Figura 1. Mecanismos de acción propuestos del plasma convaleciente en enfermedad por coronavirus 2019 (COVID-19). Ac: anticuerpos; IgIV: inmunoglobulina intravenosa.

8.18-8.95\%), siendo mayor entre los pacientes críticos, incluidos los pacientes ingresados en la unidad de terapia intensiva vs. los no admitidos en la unidad de terapia intensiva (10.5 vs. $6.0 \%$ ), ventilado mecánicamente vs. no ventilado mecánicamente ( 12.1 vs. $6.2 \%)$ y aquellos con shock séptico o disfunción/falla de múltiples órganos vs. shock séptico o disfunción/falla de múltiples órganos (14.0 vs. $7.6 \%$ ). La intención a priori era crear un grupo de control de comparación para determinar la eficacia potencial usando pacientes hospitalizados con infecciones por COVID-19 durante el mismo periodo de tiempo. Esta decisión se tomó después de la colaboración con la FDA, por lo cual este informe es solo sobre la seguridad del plasma convaleciente para los 20,000 sujetos iniciales. Una publicación futura discutirá la eficacia potencial. Estos datos actualizados proporcionan evidencia sólida de que la transfusión de plasma convaleciente es segura en pacientes hospitalizados con COVID-19, y respaldan la noción de que la administración temprana de plasma dentro del curso clínico de COVID-19 es más probable que reduzca la mortalidad.

\section{Principios biológicos y mecanismo de acción del plasma convaleciente}

El uso de plasma convaleciente en un paciente con COVID-19 puede tener distintos efectos benéficos ${ }^{34}$, siendo el más importante la supresión de la viremia. En la infección por SARS-CoV-2 se espera el pico de la viremia en la primera semana de la infección y el desarrollo de la respuesta inmunitaria primaria hacia el día 10 a 14, que da paso al aclaramiento viral. Por lo anterior, se infiere que la utilidad de la administración de plasma convaleciente es mayor durante las fases tempranas de la enfermedad. Además de la neutralización se han descrito otros potenciales mecanismos de acción (Fig. 1).

\section{Neutralización viral mediada por anticuerpos}

Dado que el SARS-CoV-2 utiliza el receptor de la enzima convertidora de angiotensina 2 (ACE2) en la superficie de la célula huésped para unirse y entrar a ella mediante su proteína Spike (S), este resulta una diana atractiva para prevenir la captación viral. Entre las opciones terapéuticas posibles está el uso de anticuerpos neutralizantes de suero convaleciente o el uso de anticuerpos diseñados por ingeniería molecular. Estos pueden tener diversos mecanismos de acción según su diseño u origen, como el dominio de unión al receptor soluble (basado en la proteína SAR-S) que ocuparía el receptor de la ACE2 o su forma soluble evitando el acceso al SARS-CoV-2 o bien secuestrando de manera competitiva al receptor de la ACE2 unido a la superficie celular en las células huésped, evitando de la misma manera la captación viral ${ }^{35}$.

\section{Citotoxicidad celular mediada por anticuerpos, activación del complemento y fagocitosis}

La presencia de anticuerpos contra el virus en cuestión puede suprimir la viremia mediante 
mecanismos neutralizadores, proporcionando una inmunidad inmediata, aunque transitoria, al huésped susceptible, permitiendo además el desarrollo de inmunidad activa posterior a la unión de la porción Fc (presente en anticuerpos de ocurrencia natural y los diseñados por ingeniería molecular), facilitando la presentación de antígenos por células dendríticas y linfocitos $\mathrm{T}$ helper (citotoxicidad mediada por anticuerpos), la activación del complemento y la fagocitosis; estudios in vitro sugieren también un efecto benéfico planteando que la inmunidad pasiva favorece el aclaramiento de células infectadas ${ }^{35,36}$.

\section{Inmunomodulación}

La presencia de anticuerpos no neutralizantes con capacidad de unión viral puede jugar un papel importante. Se ha propuesto que además de los mecanismos descritos esta variedad de anticuerpos tengan un efecto inmunomodulador, el cual podría limitar el daño tisular mediado por la respuesta inflamatoria sistémica del huésped en la fase temprana de la infección ${ }^{36-38}$.

La mayoría de los estudios describen su uso terapéutico en una dosis única de plasma convaleciente obtenido de 1 o 2 donadores (esto último, de forma teórica, podría incrementar la eficacia de la intervención al obtener anticuerpos de calidad y capacidad neutralizante distintos y eventualmente dirigidos a distintos epítopos) ${ }^{38}$. Se ha propuesto el uso de profilaxis en el contexto de individuos susceptibles y personal de salud que se encuentre en exposición constante a la infección, aunque no se tiene aprobación local ni internacional.

\section{Eventos adversos a la transfusión de plasma convaleciente}

El uso terapéutico de plasma convaleciente como modalidad de inmunidad pasiva puede provocar eventos adversos los cuales pueden ser clasificados en dos grupos: conocidos y teóricos.

\section{Riesgos conocidos}

\section{TRANSMISIÓN DE INFECCIONES}

Los riesgos conocidos asociados a la transfusión de cualquier hemocomponente son aquellos inherentes a la transmisión de agentes infecciosos (virus de la inmunodeficiencia humana, virus de la hepatitis $B$ y $C$, sífilis y enfermedad de Chagas, entre otros). Con el advenimiento de técnicas de detección de agentes transmitidos por transfusión (serología y técnicas de amplificación de ácidos nucleicos) el riesgo de la transmisión inadvertida de patógenos o agentes infecciosos susceptibles de generar una reacción adversa a la transfusión es muy bajo.

\section{REACCIONES SECUNDARIAS A LOS CONSTITUYENTES DEL PLASMA \\ Lesión pulmonar aguda asociada a la transfusión}

Es importante recalcar que el uso de suero convaleciente se realizará en pacientes con condiciones predisponentes, particularmente afectación pulmonar secundaria a COVID-19 lo cual aumentará su susceptibilidad a eventos adversos por lesión pulmonar aguda (LPA) asociada a transfusión (TRALI, por sus siglas en inglés) ${ }^{39}$. Tomando en cuenta la teoría de los dos eventos para explicar la fisiopatología de la LPA asociada a transfusión partiremos de una fase de preactivación de polimorfonucleares (primer evento) en respuesta a la patología pulmonar predisponente (COVID-19) con lo cual moléculas de adhesión como el ligando glucoproteico-1 de la selectina $P$ y la molécula de adhesión intercelular-1 en neutrófilos, selectina $P$ y $\beta 2$-integrina en células endoteliales y selectina $P$ en las plaquetas se sobreexpresarán y promoverán interacciones celulares, induciendo la migración de neutrófilos al espacio alveolar. El denominado segundo evento constituye la transfusión de componentes que contengan factores como anticuerpos y modificadores de la respuesta biológica (tal es el caso del plasma convaleciente), con lo cual se acentuará la leucostasis pulmonar, la activación de neutrófilos y la formación de NETosis. Todo ello se traducirá en una dificultad respiratoria aguda y edema pulmonar rico en proteínas característico de la TRALI. Para minimizar dicho riesgo es fundamental que en el reclutamiento de donadores se realice cribado para la detección de anticuerpos anti-HLA (antígeno leucocitario humano) en mujeres con antecedente gestacional o bien, de no contar con dicha herramienta, descartar mujeres con antecedente gestacional de la donación ${ }^{33}$.

\section{Sobrecarga de volumen asociada a transfusión}

Es una complicación que debe tomarse en cuenta, debido a que puede coexistir con TRALI y los pacientes 
candidatos a uso de plasma convaleciente generalmente se encuentran en estado crítico, con comorbilidades predisponentes y mal manejo de fluidos, lo que condicionará per se sobrecarga a ventrículo derecho aumentando el riesgo de dicho evento. Podrá prevenirse la sobrecarga de volumen con un manejo juicioso de la velocidad de infusión y uso de diuréticos.

\section{Riesgos teóricos}

\section{EFECTO POTENCIADOR VIRAL DEPENDIENTE DE ANTICUERPOS Y ANERGIA SECUNDARIA}

Existe el riesgo teórico de un efecto potenciador viral dependiente de anticuerpos, lo cual se ha explicado como complicación en otras infecciones virales o en el uso de anticuerpos monoclonales (palivizumab). Este efecto se explica debido a que al haber una infección primaria que culmina con la producción de anticuerpos subneutralizantes o no neutralizantes estos no serán capaces de proteger al huésped de una infección ulterior, pero sí facilitarán la unión de partículas virales a la célula a través de su receptor Fc. Así pues, el riesgo de infundir anticuerpos no neutralizantes o subneutralizantes se traduciría en un aumento en la captación viral y la gravedad de la infección, sin embargo, este efecto no se espera que sea de relevancia clínica en el contexto de anticuerpos policlonales (como los existentes en el plasma convaleciente) y la evidencia disponible sugiere la seguridad de esta intervención terapéutica ${ }^{40}$. Otro riesgo teórico propuesto es la atenuación que podría tener sobre el sistema inmunitario del receptor el administrar una carga de anticuerpos de manera pasiva, por lo que se sugeriría la inmunización sistemática de estos individuos al contar con una vacuna disponible para SARS-CoV-2 ${ }^{35}$.

\section{Aumento del Riesgo tRombótico}

Las alteraciones hematológicas, específicamente en lo que respecta a la coagulación, han sido descritas en otro apartado de este suplemento, sin embargo vale la pena recalcar que el paciente con infección por SARS-CoV-2 manifestará datos clínicos de una coagulopatía intravascular pulmonar mediada principalmente por mecanismos inmunotrombóticos, la cual se traduce en elevación del fibrinógeno y dímero $\mathrm{D}$ y una activación importante del sistema del plasminógeno-plasmina ${ }^{41}$, como reflejo de un daño alveolar difuso e inflamación endotelial, con un efecto dominó potenciado por una desregulación de la respuesta endotelial pulmonar y posteriormente una coagulación intravascular diseminada, teniendo como desenlace trombosis (raramente hemorragia) ${ }^{42}$. Indudablemente un paciente con factores de riesgo y dichas alteraciones en la coagulación tendrá que someterse a un esquema de tromboprofilaxis como parte integral del manejo. Actualmente no existe evidencia de que el uso de plasma convaleciente aumente el riesgo tromboembólico del paciente, probablemente el uso de este componente (que contiene factores procoagulantes) se vea atenuado por el efecto inmunomodulador de los elementos que contiene (anticuerpos no neutralizantes, citocinas antiinflamatorias, factores anticoagulantes [proteína C y S]).

Existe evidencia disponible plasmada en la experiencia del EAP (Joyner, et al., Mayo Clinic, previamente mencionado), quienes reportan una incidencia baja de eventos adversos graves. De un universo de 20,000 pacientes, solo 89 tuvieron reacciones transfusionales (< 1\%), 87 eventos tromboembólicos 0 trombóticos (< 1\%), 680 eventos cardiovasculares ( 3\%). En estos dos últimos rubros la mayor parte de los eventos no fueron atribuidos a la transfusión per se (55 y 562 eventos no relacionados a la transfusión respectivamente) $)^{38}$.

\section{Selección del potencial donador de plasma convaleciente}

La obtención de plasma convaleciente debe realizarse solo en individuos que cumplan de manera estricta con la normativa local vigente. Las guías internacionales, destacando la emitida por FDA, destacan cuatro puntos clave: evidencia documentada por laboratorio de infección por SARS-CoV-2, resolución completa de los síntomas, sexo del donador y presencia de anticuerpos neutralizantes (si se cuenta con ella). En las figuras 2 y 3 se muestra un algoritmo propuesto para la selección del donador recuperado de la infección por SARS-CoV-2 para la donación de plasma convaleciente.

\section{Prueba confirmatoria}

Se debe tener evidencia laboratorial que confirme la enfermedad: reacción en cadena de la polimerasa por exudado nasofaríngeo u orofaríngeo o una prueba serológica positiva en caso de no contar con prueba confirmatoria cuando se sospechó de COVID-19. 


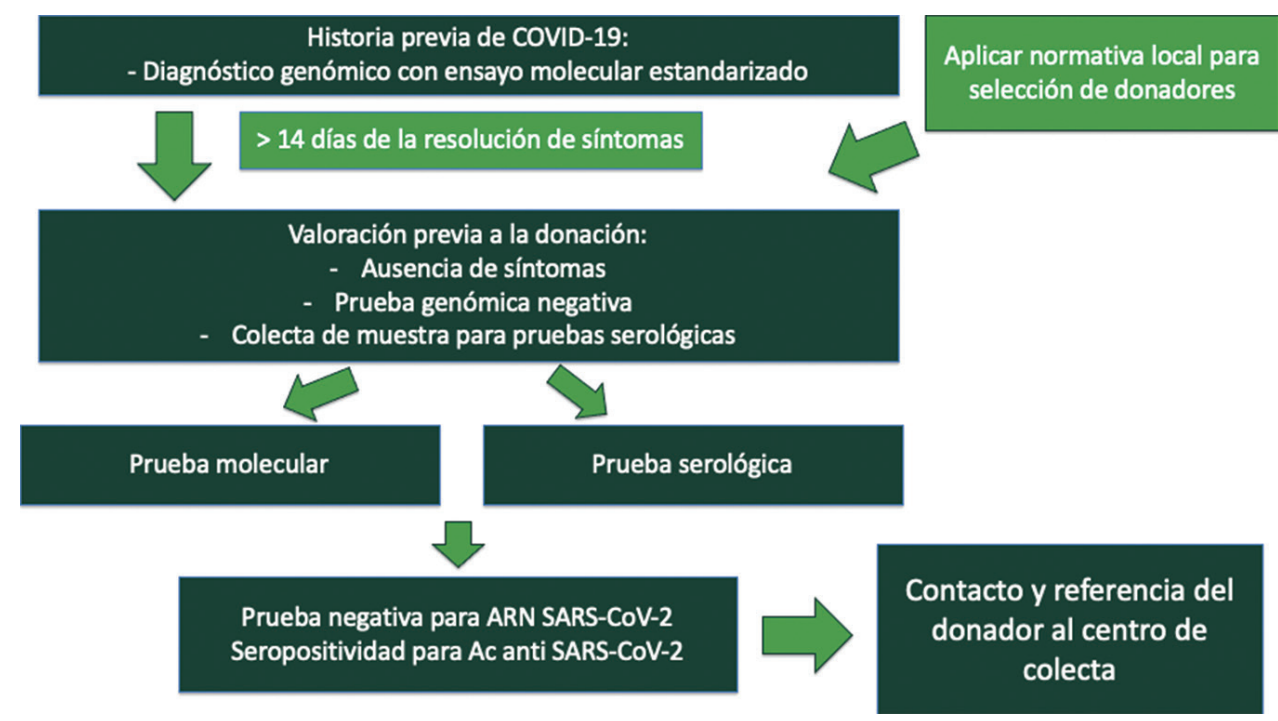

Figura 2. Algoritmo sugerido para la preselección de donadores de plasma convaleciente en enfermedad por coronavirus 2019 (COVID-19). Ac: anticuerpos; SARS-CoV-2: coronavirus 2 del síndrome respiratorio agudo grave.

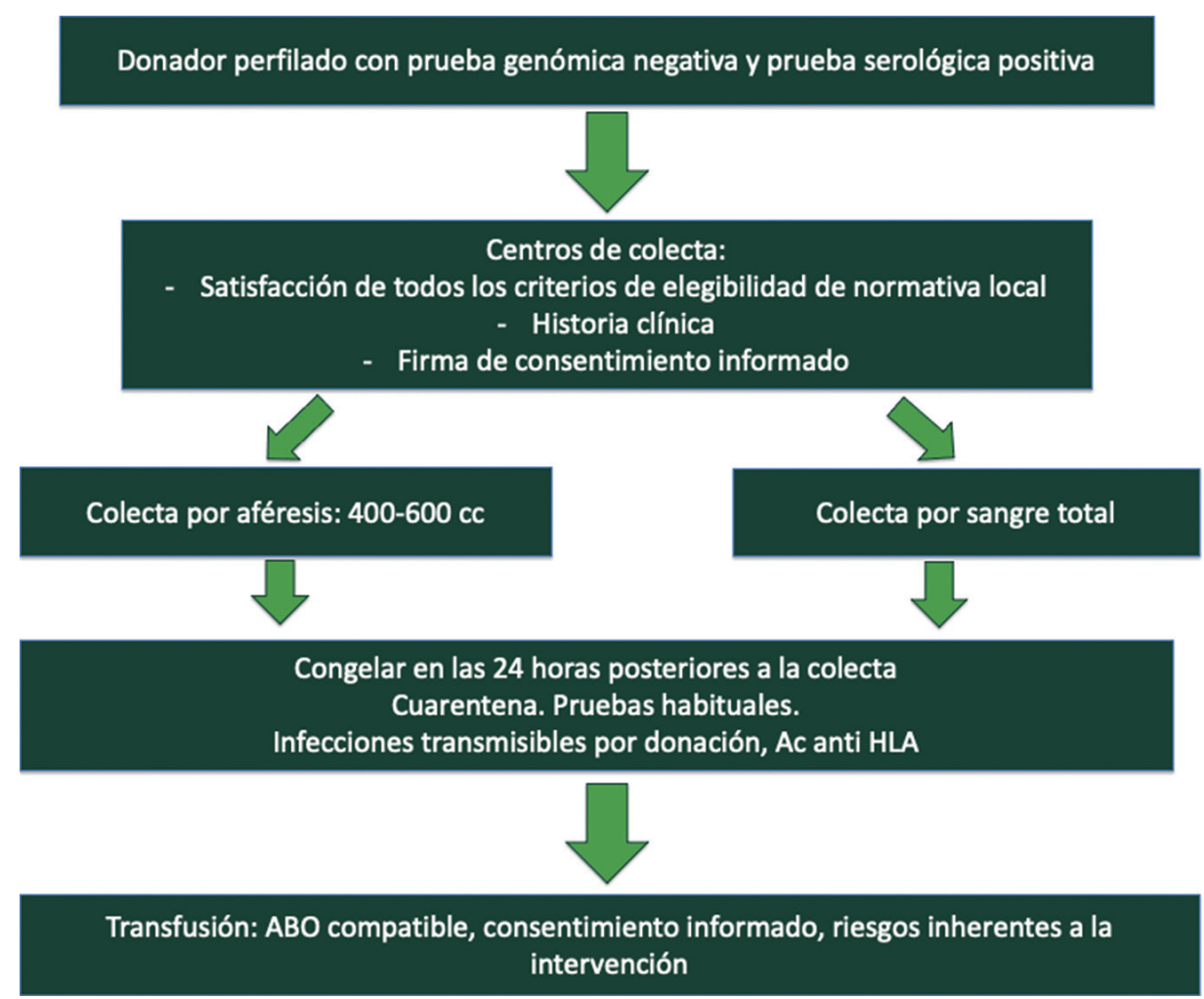

Figura 3. Algoritmo sugerido para el proceso de obtención y administración de plasma convaleciente en enfermedad por coronavirus 2019 (COVID-19).

Ac: anticuerpos; HLA: antígeno leucocitario humano.

\section{Resolución completa de la sintomatología}

A pesar de que la respuesta inmunológica en la infección por SARS-CoV-2 no es conocida del todo y los valores clínicos representativos para el escrutinio de anticuerpos no han sido establecidos, se cuenta con estudios pivotales que nos dan un panorama general del comportamiento serológico de la enfermedad. 
Un estudio en China que enroló a 173 pacientes demostró una tasa de seroconversión para anticuerpos totales, inmunoglobulina (Ig) M e IgG del 93, 82.7 y $64.7 \%$ en una media de 11,12 y 14 días, respectivamente. La tasa de producción de anticuerpos incrementa rápidamente de $<40 \%$ antes del día 7 del inicio de la enfermedad hasta el 100, 94.3 y $79.8 \%$ para anticuerpos totales, $\lg M$ e $\lg G$ respectivamente hacia el día 15 de la enfermedad. A la par se espera una tasa de aclaramiento de ARN del $66.7 \%$ antes del día 7 hasta un $45.5 \%$ después del día 15 . No se encontraron diferencias en la tasa de formación de anticuerpos haciendo el comparativo entre pacientes con infección clínicamente leve e infección grave ${ }^{43}$. En un estudio italiano el $67 \%$ de un total 60 donadores voluntarios de sangre total provenientes de la provincia de Castiglione d'Adda tuvieron una conversión serológica a pesar de haber cursado asintomáticos desde el diagnóstico genómico de la enfermedad ${ }^{44}$. Es por ello que las guías implementadas de manera internacional (AABB [antiguamente American Association of Blood Banks], FDA, EBA [European Blood Alliance]) recomiendan el reclutamiento de donadores hacia el día 14 del fin de la sintomatología. De manera deseable los donadores deben contar con una prueba de reacción en cadena de la polimerasa negativa para garantizar la seguridad del personal involucrado en el proceso de selección del donador y obtención de plasma convaleciente.

\section{Sexo del donador}

Se consideran candidatos donadores masculinos 0 femeninos sin antecedentes gestacionales. Si se desea incluir donadoras con antecedente gestacional es necesario un escrutinio negativo para anticuerpos antiHLA (riesgo de TRALI, ver apartado de eventos adversos a la transfusión de plasma convaleciente) ${ }^{33}$.

\section{Titulación de anticuerpos neutralizantes}

Respecto a la titulación de anticuerpos totales, título de lgG o IgM previos a la colecta de plasma, no existe evidencia que correlacione con la capacidad de neutralización; si se cuenta con la capacidad para medir anticuerpos neutralizantes es recomendable que el predonador tenga un título óptimo de anticuerpos antiSARC-CoV-2 (> 160), un título de 1:80 se considera aceptable si un se cuenta con otra unidad compatible $^{45}$. Se propone adicionalmente la realización de ensayos de neutralización, los cuales involucran el uso de cultivos celulares, siendo óptima una cifra $>40$, cabe destacar que la complejidad de estos ensayos y la necesidad de contar con un laboratorio con nivel de bioseguridad III limita mucho su aplicación en la práctica diaria, por lo que su uso no se considera obligatorio. En caso de no contar con la capacidad de realizar ninguno de estos estudios se recomienda la integración de una seroteca para su determinación ulterior.

\section{Selección de pacientes candidatos a recibir plasma convaleciente}

De acuerdo con lineamientos emitidos por FDA ${ }^{38,43}$, se sugiere el uso de plasma convaleciente en los siguientes contextos:

- Enfermedad grave: disnea, frecuencia respiratoria $\geq 30$ respiraciones por minuto, saturación de oxígeno en sangre $\leq 93 \%$, relación de presión parcial de oxígeno arterial/fracción de oxígeno inspirado $<300$ o infiltrados pulmonares $>50 \%$ dentro de 24 a 48 horas al ingreso.

- Enfermedad potencialmente mortal: insuficiencia respiratoria, choque séptico o disfunción o falla de múltiples órganos.

Adicional a esto se sugiere el uso en pacientes con comorbilidades o factores de riesgo; el uso de plasma convaleciente deberá estar respaldado por un ensayo clínico, apegándose a la normativa local, contando con aprobación del comité de ética y un consentimiento informado del paciente o familiar responsable.

\section{Obtención de plasma convaleciente: fraccionamiento de sangre total y plasmaféresis}

La plasmaféresis es un procedimiento de separación celular que permite la obtención de plasma de un donador y el retorno del componente celular a la circulación corporal, lo que permite la colecta de una mayor cantidad del componente deseado (plasma), una mayor calidad de este, una colecta de un mayor volumen de plasma convaleciente, una menor incidencia de eventos adversos a la donación y transfusión y la posibilidad de contar con donantes de repetición. Es posible también obtener este componente mediante fraccionamiento de sangre total, con la desventaja de limitar el número de eventos transfusionales, mayor incidencia de eventos adversos a la donación y mayor contenido celular residual en el 
componente, lo que pudiera asociarse a una mayor tasa de eventos adversos a la transfusión.

\section{Administración de plasma convaleciente}

Se sugiere la administración se realice en una infusión lenta, en un periodo no mayor a 6 horas, sin embargo, dado que la viabilidad de los anticuerpos contenidos en el producto no se compromete tanto como los factores de coagulación (termolábiles), en situaciones especiales (pacientes con sobrecarga de volumen, insuficiencia ventricular derecha) podrá extenderse el periodo de administración. No se sugiere el uso sistemático de premedicación, especialmente esteroides, los cuales pudieran atenuar la respuesta inmunitaria. Deberá realizarse un reporte de eventos adversos siguiendo la normativa y considerando estos una variable principal en el análisis de los resultados del protocolo en cuestión.

\section{Normatividad nacional}

El Lineamiento técnico para protocolos de investigación relacionados con el uso terapéutico de plasma proveniente de donadores convalecientes de COVID19 secundaria a infección por SARS-COV-2 es un documento emitido por el Centro Nacional de la Transfusión Sanguínea, con fundamento en lo establecido por el artículo 42 del Reglamento Interior de la Secretaría de Salud, con el fin de ordenar y alinear a los diferentes bancos de sangre y centros de investigación en México que han mostrado interés en el uso con fines de investigación de plasma convaleciente de donadores recuperados de infección por SARS-CoV-2 en pacientes hospitalizados con COVID-19.

En este documento se encuentran concentrados los lineamientos para poder realizar un protocolo de investigación clínica con plasma convaleciente de donadores recuperados deSARS-CoV-2yse encuentra disponibleen: https://www.gob.mx/cnts/documentos/recome ndaciones-de-abordaje-para-atender-la-pandemiade-virus-covid-19-en-los-servicios-de-sangre?idiom=es.

Para el desarrollo del protocolo se deben observar adicionalmente las consideraciones del documento $\mathrm{La}$ Bioética ante la Pandemia del COVID-19, publicado por la Comisión Nacional de Bioética, el cual se puede consultar en: https://www.gob.mx/cms/uploads/attachment/ file/544215/Pronunciamiento_Bioetica_ante_la_pandemia_del_COVID_19.pdf.

\section{Conclusiones}

EI SARS-CoV-2 ha provocado una crisis de salud global. En ausencia de una vacuna u opciones específicas de tratamiento farmacológico para la COVIDı19, el mundo ha dirigido la atención al posible uso terapéutico del plasma convaleciente.

La evidencia científica mundial actual demuestra que desde los primeros ensayos clínicos realizados, la inmunoterapia pasiva con plasma derivado de pacientes convalecientes de COVID-19 es segura ${ }^{33}$. En cuanto a la eficacia, existen varios estudios que mostraron una estancia hospitalaria más corta y una mortalidad más baja en pacientes tratados con plasma convaleciente que en aquellos que no fueron tratados con plasma convaleciente ${ }^{27-32}$, sin embargo, existe una clara necesidad de realizar estudios clínicos aleatorizados, más rigurosos, que permitan evaluar de forma objetiva la eficacia del plasma convaleciente, ya que dentro de las limitaciones de la evidencia de la mayoría de los estudios publicados se encuentra que esta es de muy baja certeza por alto riesgo de sesgo (estudios abiertos), inconsistencia, imprecisión y evidencia indirecta. Los datos de ensayos clínicos rigurosamente controlados de plasma convaleciente son pocos, lo que subraya la necesidad de evaluar su uso de manera objetiva para un rango de indicaciones y poblaciones de pacientes específicos, que puedan ser beneficiados en mayor grado de esta intervención terapéutica.

A nivel mundial y nacional, los bancos de sangre cuentan con una infraestructura robusta para realizar recolecciones y construir inventarios de plasma convaleciente para satisfacer la creciente demanda. No obstante, existen desafíos matizados, tanto regulatorios como logísticos, que abarcan la elegibilidad de los donantes, el reclutamiento de donantes, las colecciones y la transfusión misma.

Con el fin de proporcionar a los diferentes centros de investigación que han mostrado interés en el uso con fines de investigación plasma convaleciente de donadores recuperados de infección por SARS-CoV-2 en pacientes hospitalizados con COVID-19, es importante que los establecimientos de sangre estén listos para satisfacer las solicitudes de plasma inmune definiendo los requisitos aplicables a los donantes de plasma y los estándares para la preparación, calificación, almacenamiento, distribución y control del uso del producto. Este documento de posición tiene como objetivo dar recomendaciones sobre las características biológicas de una preparación de plasma de donantes convalecientes y apoyar la evaluación de 
este enfoque terapéutico en investigaciones más rigurosas.

\section{Conflicto de intereses}

Los autores manifiestan no tener conflicto de intereses alguno.

\section{Financiamiento}

La presente investigación no ha recibido ninguna beca específica de agencias de los sectores público, comercial o sin ánimo de lucro.

\section{Responsabilidades éticas}

Protección de personas y animales. Los autores declaran que para esta investigación no se han realizado experimentos en seres humanos ni en animales.

Confidencialidad de los datos. Los autores declaran que han seguido los protocolos de su centro de trabajo sobre la publicación de datos de pacientes.

Derecho a la privacidad y consentimiento informado. Los autores declaran que en este artículo no aparecen datos de pacientes.

\section{Bibliografía}

1. Huang C,Wang Y, Li X, et al. Clinical features of patients infected with 2019 novel coronavirus in Wuhan, China. Lancet. 2020;395(10223):497-506.

2. WHO. Coronavirus disease 2019 (COVID-19) Situation Report - 155 Updated June 23, 2020. https://www.who.int/docs/default-source/coronaviruse/situation-reports/20200623-covid-19-sitrep-155.pdf?sfvrsn=ca01ebe_2.

3. Al-Tawfiq JA, Al-Homoud AH, Memish ZA. Remdesivir as a possible therapeutic option for the COVID-19. Travel Med Infect Dis. 2020 Mar 5:101615. doi: 10.1016/..tmaid.2020.101615.

4. Wang M, Cao R, Zhang L, Yang X, Liu J, Xu M, Shi Z, Hu Z, Zhong W, Xiao G. Remdesivir and chloroquine effectively inhibit the recently emerged novel coronavirus (2019-nCoV) in vitro. Cell Res. 2020 Mar;30(3):269271. doi: 10.1038/s41422-020-0282-0.

5. Dong L, Hu S, Gao J. Discovering drugs to treat coronavirus disease 2019 (COVID-19). Drug Discov Ther. 2020;14(1):58-60. doi: 10.5582/ ddt.2020.01012

6. Lu H. Drug treatment options for the 2019-new coronavirus (2019-nCoV). Biosci Trends. 2020;14(1):69-71.

7. Cortegiani A, Ingoglia G, Ippolito M, Giarratano A, Einav S. A systematic review on the efficacy and safety of chloroquine for the treatment of COVID-19. J Crit Care. 2020 Mar 10:S0883-9441(20)30390-7. doi: 10.1016/j.jcrc.2020.03.005.

8. Gautret $\mathrm{P}$, Lagier JC, Parola P, Hoang VT, Meddeb L, Mailhe M, et al. Hydroxychloroquine and azithromycin as a treatment of COVID-19: results of an open-label non-randomized clinical trial. Int J Antimicrob Agents. 2020 Mar 20:105949. doi: 10.1016/j.ijantimicag.2020.105949.

9. Lim J, Jeon S, Shin HY, Kim MJ, Seong YM, Lee WJ, Choe KW, Kang YM, Lee B, Park SJ.Case of the Index Patient Who Caused Tertiary Transmission of COVID-19 Infection in Korea: the Application of Lopinavir/Ritonavir for the Treatment of COVID-19 Infected Pneumonia Monitored by Quantitative RT-PCR. J Korean Med Sci. 2020 Feb 17;35(6):e79. doi: 10.3346/jkms.2020.35.e79.

10. Shanmugaraj B, Siriwattananon K, Wangkanont K, Phoolcharoen W. Perspectives on monoclonal antibody therapy as potential therapeutic intervention for Coronavirus disease-19 (COVID-19). Asian Pac J Allergy Immunol. 2020 Mar;38(1):10-18. doi: 10.12932/AP-200220-0773.
11. World Health Organization. WHO Blood Regulators Network (BRN) Position Paper on Use of Convalescent Plasma, Serum or Immune Globulin Concentrates as an Element in Response to an Emerging Virus* September 2017

12. Park WH. Therapeutic use of antipoliomyelitits serum in preparalytic cases of poliomyelitis. JAMA. 1932;99:1050-1053.

13. Park WH, Freeman RG. The prophylactic use of measles convalescent serum. JAMA. 1926;87(8):556-558.

14. Gallagher JR. Use of convalescent measles serum to control measles in a preparatory school. Am J Public Health Nations Health. 1935;25(5):595-598.

15. Rambar AC. Mumps; use of convalescent serum in the treatment and prophylaxis of orchitis. Am J Dis Child. 1946;71:1-13.

16. Luke TC, Casadevall A, Watowich SJ, Hoffman SL, Beigel JH, Burgess TH. Hark back: passive immunotherapy for influenza and other serious infections. Crit Care Med. 2010;38(4 suppl):e66-e73.

17. Luke TC, Kilbane EM, Jackson JL, et al. Meta-analysis: convalescent blood products for Spanish influenza pneumonia: a future $\mathrm{H} 5 \mathrm{~N} 1$ treatment? Ann Intern Med. 2006;145:599-609.

18. Kraft CS, Hewlett AL, Koepsell S, et al; Nebraska Biocontainment Unit and the Emory Serious Communicable Diseases Unit. The use of TKM100802 and convalescent plasma in 2 patients with Ebola virus disease in the United States. Clin Infect Dis. 2015;61(4):496-502.

19. van Griensven J, Edwards T, de Lamballerie X, et al; Ebola-Tx Consortium. Evaluation of convalescent plasma for Ebola virus disease in Guinea. N Engl J Med. 2016;374(1):33-42.

20. Florescu DF, Kalil AC, Hewlett AL, et al. Administration of brincidofovir and convalescent plasma in a patient with Ebola virus disease. Clin Infect Dis. 2015;61(6):969-973.

21. Zhou B, Zhong N, Guan Y. Treatment with convalescent plasma for influenza A (H5N1) infection. N Engl J Med. 2007;357(14):14501451.

22. Hung IF, To KK, Lee CK, et al. Convalescent plasma treatment reduced mortality in patients with severe pandemic influenza A (H1N1) 2009 virus infection. Clin Infect Dis. 2011;52(4):447-456.

23. Burnouf $T$, Radosevich M. Treatment of severe acute respiratory syndrome with convalescent plasma. Hong Kong Med J. 2003;9(4):309.

24. Cheng $Y$,Wong R, Soo YO, et al. Use of convalescent plasma therapy in SARS patients in Hong Kong. Eur J Clin Microbiol Infect Dis. 2005;24 (1):44-46.

25. Soo YO, Cheng $Y$, Wong $R$ et al. Retrospective comparison of convalescent plasma with continuing high-dose methylprednisolone treatment in SARS patients. Clin Microbiol Infect. 2004; 10: 676-678.

26. Arabi $\mathrm{Y}$, Balkhy $\mathrm{H}$, Hajeer $\mathrm{AH}$, et al. Feasibility, safety, clinical, and laboratory effects of convalescent plasma therapy for patients with Middle East respiratory syndrome coronavirus infection: a study protocol. Springerplus. 2015;4:709.

27. Chen L, Xiong J, Bao L, Shi Y. Convalescent plasma as a potential therapy for COVID-19. Lancet Infect Dis. 2020;S1473-3099(20)301419.

28. Binzhen Chen a, Rong Xia. Early experience with convalescent plasma as immunotherapy for COVID-19 in China: Knowns and unknowns. Vox Sang 2020 Jun 9;10.1111/vox.12968.doi: 10.1111/vox.12968

29. Shen $C$, Wang Z, Zhao F, et al.: Treatment of 5 critically ill patients with COVID-19 with convalescent plasma. JAMA 2020.

30. Duan K, Liu B, Li C, et al.: Effectiveness of convalescent plasma therapy in severe COVID-19 patients. Proc Natl Acad Sci USA 2020

31. Zhang B, Liu S, Tan T, et al.: Treatment with convalescent plasma for critically ill patients with SARS-CoV-2 infection. Chest 2020.

32. Ling Li, Wei Zhang, et al. Effect of Convalescent Plasma Therapy on Time to Clinical Improvement in Patients With Severe and Life-threatening COVID-19. A Randomized Clinical Trial. JAMA. Published online June 3, 2020. doi:10.1001/jama.2020.10044.

33. Joyner MJ, Bruno KA, et al. Mayo Clinic Proceedings: Safety Update: COVID-19 Convalescent Plasma in 20,000 Hospitalized Patients. En: https://els-jbs-prod-cdn.jbs.elsevierhealth.com/pb/assets/raw/Health\%20 Advance/journals/jmcp/jmcp_ft95_6_8.pdf

34. Zhang L, Liu Y. Potential interventions for novel coronavirus in China: A systematic review. J Med Virol. 2020;92(5):479-490.

35. Casadevall A, Pirofski LA. The convalescent sera option for containing COVID-19. J Clin Invest. 2020.

36. Casadevall A, Pirofski LA. Antibody-mediated regulation of cellular immunity and the inflammatory response. Trends Immunol. 2003;24(9):474-478.

37. Bloch EM, Shoham S, Casadevall A, et al. Deployment of convalescent plasma for the prevention and treatment of COVID-19. J Clin Invest. 2020 April 7.

38. Tiberghien $\mathrm{P}$, de Lambalerie $\mathrm{X}$, Morel $\mathrm{P}$, et al. Collecting and evaluating convalescent plasma for COVID-19 treatment: why and how. Vox Sang. 2020 April 2. [Epub ahead of print].

39. Gajic $\mathrm{O}$, et al. Transfusion-related acute lung injury in the critically ill: prospective nested case-control study. Am J Respir Crit Care Med. 2007;176(9):886-891. 
Gaceta Médica de México. 2021;157(Supl 3)

40. Wan $Y$, et al. Molecular mechanism for antibody-dependent enhancement of coronavirus entry. J Virol. 2020;94(5):e02015-19.

41. Ji, H. L., Zhao, R., Matalon, S., \& Matthay, M. A. Elevated plasmin (ogen) as a common risk factor for COVID-19 susceptibility. Physiological Reviews, 2020, 100(3), 1065-1075

42. Connors, J. M., \& Levy, J. H. COVID-19 and its implications for thrombosis and anticoagulation. Blood. 2020

43. Zhao, J., Yuan, Q., Wang, H., Liu, W. et al. Antibody responses to SARSCoV-2 in patients of novel coronavirus disease 2019. Clinical Infectious Diseases. 2020.
44. Serra M. Coronavirus, Castiglione d'Adda è un caso di studio: "II $70 \%$ dei donatori di sangue è positivo" [article in Italian]. 2020; https://www. lastampa.it/topnews/primo-piano/2020/04/02/news/coronavirus-castiglione-d-adda-e-un-caso-di-studio-il-70-dei-donatori-di-sangue-epositivo-1.38666481. Accessed April 08, 2020.

45. Center for Biologics Evaluation and Research. Revised Information for Investigational COVID19 Convalescent Plasma. http://www.fda.gov/ vaccines-blood-biologics/investigationalnew-drug-ind-or-device-exemption-ideprocess-cber/investigational-covid-19- convalescent-plasma-emergency-inds. 